\title{
Editorial
}

\section{Digital libraries as experienced by the editors of the journal}

\author{
Erich Neuhold, Ingo Frommholz
}

Published online: 27 July 2004 - (C) Springer-Verlag 2004

Due to the relaunch of the Journal of Digital Libraries and the introduction of a new editorial board, the editors were asked to write about their experiences and project results in the target field of the journal, which is digital libraries. The submitted articles are compiled in this special issue of the Journal of Digital Libraries, which is entitled 'Digital libraries as experienced by the editors of the journal'. All editorial board members have been involved in this issue - either by reporting about their projects or by reviewing the submitted articles.

This special issue reveals the rich variety and facets of methods, projects, groups and institutions in the field of digital libraries. The editors of the journal are involved in the development of digital library technologies and running projects or groups related to digital libraries. They see digital libraries from either a technical or an organizational perspective, which is reflected in the different kinds of articles present in this issue, describing projects and groups on the one hand and advanced methods on the other hand.

The projects, groups and institutions described here cover a broad range of possible applications. Many of them are located in the area of cultural heritage, which has become important in the context of digital libraries. Two examples of cultural heritage projects, the European Union funded projects ECHO and COLLATE, are presented in this issue, dealing with historic film archives and material, respectively. Other institutions taking part in the digitization, management and publishing of cultural assets are the John von Neumann Digital Library (dealing with Hungarian cultural heritage material) and the National Digital Library of Portugal (BND). The latter serves as an example of how traditional national libraries are concerned with their role in the digital world, trying to combine new possibilities offered by digital means with the classical approaches national libraries have been following for centuries.

Another interesting tendency that can be seen is motivated by the idea that digital libraries should not only present information coming from a single provider like an archive or museum, but also let users become aware of each other. In this way the users benefit from sharing each others' knowledge or the results of their work with the material. Collaborative working environments supporting this have been created within the COLLATE and CYCLADES projects, where users have the possibility to, for example, annotate objects and share these annotations with other users. A context-based method like the one developed within COLLATE can employ such annotations for document retrieval. Collaborative authoring of paths such as used in the Walden's Path project can support information discovery as well. Information stored in a digital repository or even on the World Wide Web can be employed to create different information structures used in a specific context by setting up a path between documents to follow when looking for specific information.

There is a strong relationship between knowledge and libraries. Material stored in libraries contains knowledge and to make this material available is the primary aim of a digital library. Knowledge extracted from documents is used to create new knowledge. This makes digital libraries interesting for areas such as knowledge management. To apply digital library technology to knowledge management in a qualification management framework is the goal of the e-Qualification project.

Since the material at hand is not necessarily only textual, multimedia approaches are gaining importance for digital libraries either for educational purposes or by dealing with multimedia archival material such as historic films. Some of the projects presented here are concerned with the integration of multimedia objects into digital 
libraries. The Open Video Digital Library, for example, is a project aiming to provide digital open source video content to research teams and educational communities, as well as serving as a test bed for video information retrieval. A system supporting educational communities is LectureLounge, which is used for capturing, managing and publishing presentations on video. The Físchlár system supports indexing, browsing and searching digital video material. Applied in the area of historic film archives, one of the ECHO project's goals is to develop services for assisting the indexing and retrieval of audiovideo documentaries.

Digital libraries are more than the migration of traditional library functionality into a digital environment. There are several new requirements coming from the document types to be handled (textual and multimedia) as well as regarding issues such as collaboration. OpenDLib is an innovative system addressing these requirements, consisting of a set of digital library services and a customizable architectural infrastructure. A new document model, DoMDL, is supported in this framework.

Getting away from the more technical aspects of digital libraries described above, it is essential that systems consider the actual information needs their users have. Collaborative working environments are trying to meet such requirements. Nevertheless, a lot of research and evaluation has to be performed; therefore, groups like telos at the Darmstadt University of Technology and the Hungarian John von Neumann Digital Library are concerned with a more user-oriented view of developments in the digital library field.

Besides presenting their projects, groups or institutions, the editors of the Journal of Digital Libraries also dis- cuss technological issues which are important to realize the variety of goals of digital libraries. These issues are:

- Metadata mapping: there exists a large amount of different metadata 'standards' underlying digital library systems. To be able to keep track of changes of these standards as well as to enable interoperability, techniques for schema mapping have to be developed, like the one described in this issue by Pentaris and Ioannidis.

- Metadata creation and enrichment: to support location, presentation and retrieval of documents, text mining methods can be applied on a document collection, enriching the collection with high-quality metadata. Witten et al. describe a way to enhance the Greenstone digital library system with GATE, a development environment for text mining.

- Personalization is another important topic which can help a system to better match users' information needs and to improve information access. In their article, Koutrica and Ioannidis discuss query personalization in digital libraries, offering a rule-based approach focusing on structured queries involving metadata in relational databases.

- Aesthetic hypermedia presentation: the content of digital museums can be published in the form of museum exhibitions based on hypermedia documents. Style elements are used to support the presentation of the content. Hong et al. present their work in this area, discussing a digital museum exhibition management framework supporting the production of an exhibition from the digital archive, the production of an exhibition site and the creation of different presentation styles for different users. 